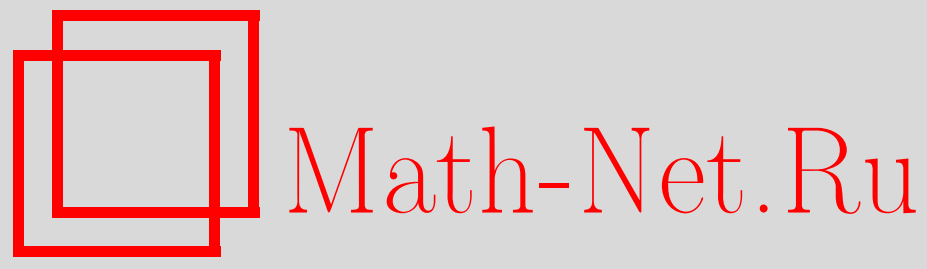

G. Kerkyacharian, O. V. Lepskiı̌, D. Picard, Nonlinear estimation in anisotropic multiindex denoising. Sparse case, Теория вероятн. и ее примен., 2007, том 52, выпуск 1, 150-171

DOI: https://doi.org/10.4213/tvp9

Использование Общероссийского математического портала MathNet.Ru подразумевает, что вы прочитали и согласны с пользовательским соглашением

http: //www . mathnet.ru/rus/agreement

Параметры загрузки:

IP : 3.85 .183 .62

26 апреля 2023 г., 18:27:31

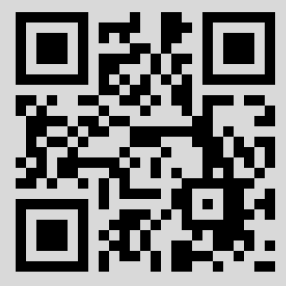




\title{
NONLINEAR ESTIMATION IN ANISOTROPIC MULTIINDEX DENOISING. SPARSE CASE ${ }^{1)}$
}

\begin{abstract}
Известно, что в размерности 1 сушествует лишь два (не считая граничного случая) типа асимптотики минимаксного риска, определенного на классе Бесова. В зависимости от соотношения между параметрами класса и нормой, описывающей риск, различаются плотный и разреженный случаи.

В нашей недавней работе [5] в задаче оценивания на анизотропном классе Бесова мы для произвольной размерности $d \geqslant 1$ получили результаты, аналогичные плотному случаю при $d=1$. Настоящая работа посвящена той же проблематике, но уже для других соотношений между используемыми параметрами. В частности, открытым оставался вопрос о связи размерности и количества различных типов скоростей сходимости. Мы покажем, что ответ не зависит от размерности, т.е., как и в размерности 1 , сушествует только два режима асимптотики минимаксного риска плотный и разреженный, за исключением границы, где асимптотика (точная) пока не найдена.

Стоит заметить, что наша процедура оценивания допускает такой выбор параметров, при которых она является адаптивной с точностью до логарифмического множителя в плотном случае (см. [5]) и минимаксно адаптивной в разреженном случае. Интересно также, что в разреженном случае свойства вложения пространств являются фундаментальными.
\end{abstract}

Ключевые слова и фразы: непараметрическое оценивание, устранение шума, анизотропная гладкость, минимаксная скорость сходимости, анизотропные пространства Бесова.

\section{Introduction}

Our aim in this paper is to complete the study initiated in the former paper [5]. In that paper, we provided a procedure of nonparametric denoising constructed on a pointwise kernel estimation with a local selection of the multidimensional bandwidth parameter. More precisely our model was and still will be

$$
X_{\varepsilon}(d t)=f(t) d t+\varepsilon W(d t), \quad t=\left(t_{1}, \ldots, t_{d}\right) \in \mathscr{D} .
$$

* Laboratoire d'Analyse, Topologie et Probabilités, Université Aix-Marseille 1, 39, rue F. Joliot-Curie, 13453 Marseille Cedex 13, France; e-mail: Oleg.Lepski@cmi.univ-mrs.fr 
( $d=2$ is precisely the case of an image with an additional noise.) Since we will not discuss special boundary effect, $\mathscr{D}$ will be chosen equal to $[0,1]^{d}$.

We consider a kernel estimation of the form

$$
\hat{f}_{\varepsilon}(h, t)=\int_{\mathscr{D}} \frac{1}{h_{1} \cdots h_{d}} K\left(\frac{x_{1}-t_{1}}{h_{1}}, \ldots, \frac{x_{d}-t_{d}}{h_{d}}\right) X_{\varepsilon}(d x),
$$

$K$ is a kernel with good approximation properties in each direction and our procedure provides a selector of the multidimensional parameter $h=$ $\left(h_{1}, \ldots, h_{d}\right)$ depending on the point $t \in \mathscr{D}$ and using the data $X_{\varepsilon}$.

1.1. Motivation. In this section we would like to discuss briefly the motivations of this paper. The main goal here is to show how to build the minimax theory (i.e., to prove the theoretical optimality of the method proposed) using a so-called «oracle» type result. Let us mention that this approach can be applied to any «oracle», but here we restrict ourselves to the consideration of the «oracle» based on the family of kernel estimators $\mathscr{K}=\left(\hat{f}_{\varepsilon}(h, \cdot), h \in(0,1]^{d}\right)$. We also point out that in this section we want to present only the «philosophy» of our approach. So we do not give here the exact definitions and try to avoid the mathematical precisions which will be given later.

1.1.1. «Oracle» inequalities. Let $\kappa=\left(\kappa_{1}, \ldots, \kappa_{d}\right) \in(0,1]^{d}$ be a given vector. Suppose that, among all the multidimensional bandwidths $h=\left(h_{1}, \ldots, h_{d}\right)$ there exists an «ideal» multibandwidth, say, $h_{\varepsilon}(f, t, \kappa)=$ $\left(h_{\varepsilon}^{1}(f, t, \kappa), \ldots, h_{\varepsilon}^{d}(f, t, \kappa)\right)$, which depends on the underlying function $f$, the point $t \in \mathscr{D}$, the noise level $\varepsilon$, and the vector $\kappa$. Let us consider the «pseudo»estimator $\hat{f}_{\varepsilon}\left(h_{\varepsilon}(f, \cdot, \kappa), \cdot\right) \in \mathscr{K}$ (we call it «pseudo»-estimator because of the dependence on the unknown function $f$ ). The «ideal choice» (properly defined in [5]) refers to the fact that in order to estimate the known function $f$ at a point $t$ we could seek for an optimal choice in the family $\mathscr{K}$ with the additional restriction $h \in(0, \kappa]$. For any underlying function $f$ at any point $t$ let us define the pointwise risk of the «pseudo»-estimator $\hat{f}_{\varepsilon}\left(h_{\varepsilon}(f, t, \kappa), t\right)$ by

$$
R_{\varepsilon}^{(p)}(f, t, \kappa)=\mathbf{E}_{f}\left|\hat{f}_{\varepsilon}\left(h_{\varepsilon}(f, t, \kappa), t\right)-f(t)\right|^{p}, \quad p>0 .
$$

This pointwise risk is usually called an «oracle» and the goal is to construct an estimator, say, $f_{\varepsilon}^{*}(\cdot, \kappa)$ which «attains» the «oracle», i.e., for all $f, t$, and $\kappa$

$$
\mathbf{E}_{f}\left|f_{\varepsilon}^{*}(t, \kappa)-f(t)\right|^{p} \leqslant C(p, d) R_{\varepsilon}^{(p)}(f, t, \kappa),
$$

where $C(p, d)$ is a constant independent of the function $f$, the noise level $\varepsilon$, the point $t$, and the vector $\kappa$.

Such an inequality (3) is called a pointwise «oracle» inequality.

In our former paper [5] we proposed the construction of an estimator $f_{\varepsilon}^{*}(\cdot, \kappa)$ and proved an «oracle» inequality $(3)$. Our construction leads to the kernel estimator $\hat{f}_{\varepsilon}\left(\bar{h}_{\varepsilon}(\cdot, \kappa), \cdot\right)$ with a random multibandwidth $\bar{h}_{\varepsilon}(t, \kappa)$ which depends on the point $t$, on the vector $\kappa$, and on the data. Our method is a generalization of Lepski's method of adaptation [6], [7], which roughly 
consists in choosing the «coarsest» bandwidth such that the estimated bias is negligible.

Before discussing the possible applications of a pointwise «oracle» inequality let us make some remarks.

1. Our construction (leading to the estimator $\hat{f}_{\varepsilon}\left(\bar{h}_{\varepsilon}(\cdot, \kappa), \cdot\right)$ ) can be used in any statistical model, where kernel estimators make sense. However, the pointwise «oracle» inequality is a mathematical result which has to be proved in any particular case. In [5] we proved (3) for the Gaussian white noise model. As to other models we think that one can obtain a similar inequality for the density model under the assumption that the underlying density is compactly supported and bounded below by some positive constant. We also believe that a similar result can be obtained for the regression model with usual properties of the design and i.i.d. noise satisfying some moment's restrictions.

2. The vector $\kappa$ involved in the definition of the «oracle» and in the description of the estimator $\hat{f}_{\varepsilon}\left(\bar{h}_{\varepsilon}(\cdot, \kappa), \cdot\right)$ can be considered as a tuning parameter of the procedure. Its theoretical choice allows one to deduce different mathematical results from one and the same «oracle» inequality. In particular, in [5] we found evaluations of $\kappa$ for which the theoretical optimality of our method was established in some particular cases.

In this paper we do not discuss the possible applications of our procedure in practice. Let us note, nevertheless, that such a presence of tuning parameters precisely enhances the procedure allowing the statistician to vary their choice for specific applications.

3. Let $\|\cdot\|_{p}, p \geqslant 1$, be the $L_{p}$-norm on $\mathscr{D}$. Using the Fubini theorem and integrating both sides of the pointwise «oracle» inequality (3) over $\mathscr{D}$ yields the following global «oracle» inequality:

$$
\mathbf{E}_{f}\left\|f_{\varepsilon}^{*}(\cdot, \kappa)-f(\cdot)\right\|_{p}^{p} \leqslant C(p, d) \int_{\mathscr{D}} R_{\varepsilon}^{(p)}(f, t, \kappa) d t \triangleq C(p, d) R_{\varepsilon}^{(p)}(f, \kappa) .
$$

4. A global «oracle» inequality allows us also to obtain an upper bound for the estimator $\hat{f}_{\varepsilon}\left(\bar{h}_{\varepsilon}(\cdot, \kappa), \cdot\right)$ on some given set of functions. Let us suppose that $f \in \mathscr{F}$, where $\mathscr{F}$ is a set of functions (usually $\mathscr{F}$ is some compact set). The upper bound for the maximal (on $\mathscr{F}$ ) risk of our estimator follows immediately from (4):

$$
\sup _{f \in \mathscr{F}} \mathbf{E}_{f}\left\|f_{\varepsilon}^{*}(\cdot, \kappa)-f(\cdot)\right\|_{p}^{p} \leqslant C(p, d) \sup _{f \in \mathscr{F}} R_{\varepsilon}^{(p)}(f, \kappa) \triangleq C(p, d) \widetilde{R}_{\varepsilon}^{(p)}(\mathscr{F}, \kappa) .
$$

5. The description of the «oracle» as well as the pointwise «oracle» inequality requires to define the «ideal» multibandwidth $h_{\varepsilon}(f, t, \kappa)=$ $\left(h_{\varepsilon}^{1}(f, t, \kappa), \ldots, h_{\varepsilon}^{d}(f, t, \kappa)\right)$. To do it we need to assume that $f \in \mathbb{H}\left(\nu, L_{\nu}\right)$ for some $\nu>0, L_{\nu}>0$, where $\mathbb{H}\left(\nu, L_{\nu}\right)$ is an isotropic Hölder ball.

It is extremely important to notice that neither the constant $C(p, d)$ nor the construction of estimation procedure depend on $\nu$ and $L_{\nu}$. In particular, 
it allows us to establish upper bounds results on any functional space $\mathscr{F}$ such that there exist $\nu>0, L_{\nu}>0$, such that $\mathscr{F} \subseteq \mathbb{H}\left(\nu, L_{\nu}\right)$.

The belonging to $\mathbb{H}\left(\nu, L_{\nu}\right)$ is needed to prove the existence of $h_{\varepsilon}(f, t, \kappa)$ and to establish some minimal properties of its modulus of continuity.

The advantage of an «oracle» inequality is that it is established for any underlying function $f$ satisfying minimal restrictions. For example, our «oracle» inequality is proved under an assumption only a bit stronger than the continuity of the underlying function.

However, the main difficulty concerning «oracle»-type results is how to justify the «oracle» itself. In particular, our «oracle» (2) involves a proper definition of the multibandwidth $h_{\varepsilon}(f, \cdot, \kappa)$. However many objections can arise: why precisely this definition? Why are we only using kernel estimators for this construction? Are we ensured that «attaining» such an «oracle» leads to an estimation procedure which is at least reasonable?

Of course, there are some intuitive explanations of the «oracle». In particular, the coordinates of the «ideal» multibandwidth $h_{\varepsilon}(f, \cdot, \kappa)$ are chosen using a «balance» between optimal kernel approximation properties of the underlying function in each given direction and the variance of the «stochastic term» of the kernel estimator. This implies, in particular, the ability to perform some qualitative analysis of the «oracle». For example, one can say that the «oracle», i.e., right-hand side of (3) (or (4)), is «small» if the function $f$ is sufficiently smooth. One can observe also the following phenomenon: the «irregularity» of $f$ in some directions can be compensated (i.e., led to «small oracle») by its «smoothness» properties in other directions.

Sometimes the interesting properties of an «oracle» can be obtained by analyzing the proof of the corresponding «oracle» inequality. As an example let us consider briefly the so-called «oversmoothing» phenomenon.

Oversmoothing and dimensionality. Adaptive methods in general, and this kind of bandwidth selection in particular, have the reputation of suffering of a systematic oversmoothing, and there can be a fear that this phenomenon can only be worse in $d$ dimension. It happens that this is not true and we would like to discuss this fact here. First of all, let us precise what does «oversmoothing» mean. In the context of adaptive estimation based on kernel type estimators, in dimension 1 (see, for example, [10]) one can prove that if $h_{\varepsilon}(f, t, \kappa)$ is the «ideal» bandwidth, and if $\bar{h}_{\varepsilon}(t, \kappa)$ is the selected data driven bandwidth, one has the following property: for all $t, f$, and $\kappa$

$$
\bar{h}_{\varepsilon}(t, \kappa) \geqslant h_{\varepsilon}(f, t, \kappa) \quad \text { with probability tending to } 1 \text { as } \varepsilon \rightarrow 0 .
$$

The nature of this phenomenon is well known: it is connected to the use of large deviation inequalities «inherent» to adaptive estimation. 
In our paper [5] we proved that a similar phenomenon in the multivariate case occurs.

Let $h_{\varepsilon}(f, t, \kappa)=\left(h_{\varepsilon}^{1}(f, t, \kappa), \ldots, h_{\varepsilon}^{d}(f, t, \kappa)\right)$ be the «ideal» multibandwidth and let $\bar{h}_{\varepsilon}(t, \kappa)=\left(\bar{h}_{\varepsilon}^{1}(t, \kappa), \ldots, \bar{h}_{\varepsilon}^{d}(t, \kappa)\right)$ be the multibandwidth given by our method.

We proved that for all $t$, for all $\nu>0, L_{\nu}>0$, and for all $\kappa$

$$
\sup _{f \in \mathbb{H}\left(\nu, L_{\nu}\right)} \mathbf{P}_{f}\left\{\prod_{i=1}^{d} \bar{h}_{\varepsilon}^{i}(t, \kappa) \geqslant \prod_{i=1}^{d} h_{\varepsilon}^{i}(f, t, \kappa)\right\} \rightarrow 1 \quad \text { as } \quad \varepsilon \rightarrow 0 .
$$

As we see this does not necessarily imply «oversmoothing». This result shows that in some directions one can expect «oversmoothing» and in other directions «undersmoothing».

All the arguments mentioned above certainly can be considered as explanations of the «oracle» proposed. However, more serious justifications are needed.

1.1.2. How to justify an «oracle» inequality. One of the possible theoretical ways for justifying an «oracle» result is to prove the theoretical optimality of the estimation procedure «attaining» this «oracle». There are two questions to be addressed:

1) What is the criterion of optimality?

2) How is used our «oracle» inequality in this context?

Our aim is to show that there is no estimation procedure which works asymptotically better than the proposal. At the present time there exist only few criteria allowing one to compare arbitrary estimators. The minimax approach is probably the most famous one. To apply it, one has to choose a parameter space, where the maximal risk of estimators is defined. Our choice is an anisotropic Besov ball and let us briefly discuss our motivation.

(a) A lot of statistical investigation (maybe the majority of them) are devoted to the estimation of continuous functions. To develop the minimax theory in this case one needs to consider some compact subsets in the space of continuous functions. An anisotropic Besov ball $B_{\mathbf{p}, \infty}^{\mathbf{s}}(M), \mathbf{s}=\left(s_{1}, \ldots, s_{d}\right)$, $\mathbf{p}=\left(p_{1}, \ldots, p_{d}\right), M>0$, is one of the most interesting and classical examples of such a compact subset. It is rather hudge and, therefore, the assumption that the underlying function belongs to this set is not extremely artificial. It allows one to study functions with different smoothness in different directions, functions with inhomogeneous smoothness, etc. Let us mention that another strong reason for supporting anisotropy is the disparity in the nature of the coordinates which is relevant for any physical phenomenon evolving in time and space, such as, for instance, video images.

(b) Our «oracle» inequality is used in order to derive the exact asymptotic of the maximal risk of the proposed estimator on an arbitrary anisotropic Besov ball. To do that one needs to put $\mathscr{F}=B_{\mathbf{p}, \infty}^{\mathbf{s}}(M)$ in (5), 
to choose the vector $\kappa$ depending on the available information on the parameters $\mathbf{s}$ and $\mathbf{p}$, and to find the asymptotic of $\widetilde{R}_{\varepsilon}^{(p)}\left(B_{\mathbf{p}, \infty}^{\mathbf{s}}(M), \kappa\right)$.

Thus, the «oracle» inequality is a useful tool to obtain an upper bound of the maximal risk. It is well known that the minimax approach also requires to obtain a lower bound of the maximal risk of an arbitrary estimator.

1.1.3. Lower bound. The proof of the lower bound theorem follows a general type of construction for lower bounds which is used in various domains of nonparametric statistics: estimation [3], [9], adaptive estimation [8], hypotheses testing [4], [11]. This is the reason why we choose to give the construction in full generality and to obtain our lower bound result as a particular case of this construction. There is at least two advantages of this construction: firstly, it is general enough to be applied in a lot of models, secondly, one of the assumptions directly contains the rate of convergence. In the particular case of the white noise (which is the framework of this paper), our approach leads to the verification of four simple assumptions. Thus instead of giving a direct (and rather standard) proof of Theorem 2, we deduce the rate of convergence by verifying the four assumptions mentioned above.

1.1.4. Conclusion. As we have seen, the minimax theory allows one to add to qualitative analysis of the procedure «attaining» an «oracle» its theoretical properties. This can be considered as a theoretical justification the «oracle» proposed. On the other hand, the minimax theory is one of the important areas of theoretical statistics and the «oracle» inequalities represent a powerful tool for deriving minimax results.

In [5], following this «philosophy» and using the «oracle» inequalities (3) and (4) we found the minimax rate of convergence on the anisotropic Besov ball with the parameters $\mathbf{p}$ and $\mathbf{s}$ satisfying the following conditions:

$$
1-\sum_{i=1}^{d} \frac{1}{p_{i} s_{i}}>0, \quad \sum_{i=1}^{d}\left[\frac{1}{s_{i}}\left(\frac{p}{p_{i}}-1\right)\right]_{+}<2 .
$$

In this region (which, by analogy with the univariate case, can be referred to as «dense» region) the minimax rate of convergence, associated to the $L_{p}$-norm is $\varepsilon^{2 \bar{s} /(1+2 \bar{s})}$, where $\bar{s}$ is defined by $1 / \bar{s}=\sum_{i=1}^{d} 1 / s_{i}$.

In the present paper, we shall investigate the following region:

$$
1 \leqslant p_{i} \leqslant p<\infty \quad \text { for all } i, \quad 1-\sum_{i=1}^{d} \frac{1}{s_{i}} \frac{1}{p_{i}}>0, \quad \sum_{i=1}^{d} \frac{1}{s_{i}}\left(\frac{p}{p_{i}}-1\right) \geqslant 2 .
$$

This region corresponds in the one-dimensional case to the sparse case, where a minimax rate of convergence different from the dense case is observed. It was an open question to decide whether this sparse case, in the $d$-dimensional context has to be split into different regions corresponding to different minimax regimes. We will see here that the answer in negative: we still observe 
a sparse region but with a unique minimax behavior, except, as usual, on the boundary. In this region, we have the following rate of convergence:

$$
\left\{\left[\ln \varepsilon^{-1}\right]^{1 / 2} \varepsilon\right\}^{b}, \quad b=\left[1-\sum_{i=1}^{d} \frac{1}{s_{i}}\left(\frac{1}{p_{i}}-\frac{1}{p}\right)\right] p\left[1-\sum_{i=1}^{d} \frac{1}{s_{i}}\left(\frac{1}{p_{i}}-\frac{1}{2}\right)\right]^{-1}
$$

with an additional logarithmic term on the boundary. This rate of convergence coincides with the rate observed in dimension 1 (see, for instance, [12], [1], [10]). Another important remark is that the estimation procedure does not depend on the parameters $\mathbf{s}$ and $\mathbf{p}$ of the anisotropic Besov ball. It means that our estimator is not only minimax one but minimax adaptive with respect to the family of anisotropic Besov balls described by the parameters $\mathbf{s}$ and $\mathbf{p}$ belonging to the sparse region. It is worthwhile to notice that the same procedure is adaptive up to logarithmic factors in the dense zone [5].

It is also interesting to observe that in the «sparse case», the embedding properties of the spaces are fundamental, and this is the reason of our limitation to the case $1 \leqslant p_{i} \leqslant p<\infty$ since we have not been able to find embedding theorems in the literature when this condition is not valid.

1.2. Organization of the paper. The paper will be organized as follows. The second section recalls the anisotropic Besov conditions and the embedding and approximation properties which will be needed in the sequel. Then we give a rapid summary of essential tools appearing in [5]. The third section consists of two theorems. The first one states the upper bound for the risk of the procedure. The second theorem states the lower bound. In particular, the two theorems together prove that our procedure attains the minimax rate of convergence simultaneously for all values of the parameters $\mathbf{s}$ and $\mathbf{p}$ belonging to the sparse zone, in other words, it is minimax adaptive in the «sparse case». Sections 4 and 5 concern the proof of the upper bound theorem and the proof of the lower bound theorem, respectively.

\section{Anisotropic Besov balls and the results from [5]}

Let us recall the following definition of the Besov space $B_{\left(p_{1}, \ldots, p_{d}\right), \infty}^{\left(s_{1}, \ldots, s_{d}\right)}$ (see [13]).

Let $f$ be a measurable function defined on $\mathbf{R}^{d}$. For $y \in \mathbf{R}^{d}$, we define

$$
\text { for all } x \in \mathbf{R}^{d}, \quad \Delta_{y} f(x)=f(x+y)-f(x) .
$$

If $l \in \mathbf{N}$, then $\Delta_{y}^{l}$ is the $l$-iterated of the operator $\Delta_{y}$. (Of course $\Delta_{y}^{0}=I_{d}$.)

We have the following properties.

1. Let $l \in \mathbf{N}: \Delta_{y}^{l} f(x)=\sum_{j=0}^{l} C_{l}^{j}(-1)^{j+l} f(x+j y)$, especially,

$(-1)^{l+1} \Delta_{y}^{l} f(x)=\sum_{j=0}^{l} C_{l}^{j}(-1)^{j+1} f(x+j y)=\sum_{j=1}^{l} C_{l}^{j}(-1)^{j+1} f(x+j y)-f(x)$. 
2. If $k \in \mathbf{N}, m \in \mathbf{N}^{*}, 1 \leqslant p \leqslant \infty, f \in \mathbf{L}^{p}\left(\mathbf{R}^{d}\right)$, we obviously have

$$
\left\|\Delta_{y}^{k+m} f\right\|_{p} \leqslant 2^{m}\left\|\Delta_{y}^{k} f\right\|_{p}
$$

3. Less obviously, one can prove Marchaud's inequality: Let $k \in \mathbf{N}$, $m \in \mathbf{N}^{*}, 1 \leqslant p \leqslant \infty, f \in \mathbf{L}^{p}\left(\mathbf{R}^{d}\right)$, then

$$
\left\|\Delta_{y}^{k} f\right\|_{p} \leqslant a(k, m) \sum_{j=0}^{\infty}(j+1)^{m-1} 2^{-k j}\left\|\Delta_{2^{j} y}^{k+m} f\right\|_{p} .
$$

D e f i n it i o $n 1$ (inhomogeneous Besov spaces). 1. Let $e_{1}, \ldots, e_{d}$ be the canonical basis of $\mathbf{R}^{d}$. For $0<s_{i}<\infty, 1 \leqslant p_{i} \leqslant \infty$, we say that $f$ belongs to $B_{p_{i}, \infty}^{s_{i}}$ if and only if there exists $l \in \mathbf{N}, s_{i}<l$ (respectively, for all $\left.l \in \mathbf{N}, s_{i}<l\right)$ and $C\left(s_{i}, l\right)<\infty$, such that

$$
\text { for all } h \in \mathbf{R}, \quad\left\|\Delta_{h e_{i}}^{l} f\right\|_{\mathbf{L}^{p_{i}}\left(\mathbf{R}^{d}, d x\right)} \leqslant C\left(s_{i}, l\right)|h|^{s_{i}} .
$$

2. $B_{\left(p_{1}, \ldots, p_{d}\right), \infty}^{\left(s_{1}, \ldots, s_{d}\right)}=\bigcap_{i=1}^{d} B_{p_{i}, \infty}^{s_{i}}$.

$\mathrm{R}$ e $\mathrm{m}$ a r ks. 1. Thus, we are considering functions having regularity $s_{i}$ in the direction $i$ quantified in $\mathbf{L}_{p_{i}}$ in the sense mentioned above. Proposition 2 below proves that the functions having this regularity can be approximated using appropriated kernels with the rate of convergence $h^{s_{i}}$ in $\mathbf{L}_{p_{i}}$-norm.

2. The condition $\exists l \in \mathbf{N}, s_{i}<l$ can be replaced by $\forall l \in \mathbf{N}, s_{i}<l$ in such a way that one can choose indifferently an integer $l$, as soon as $l>s_{i}$.

Let us finally define the following Besov ball $B_{\mathbf{p}, \infty}^{\mathbf{s}}(M), \mathbf{s}=\left(s_{1}, \ldots, s_{d}\right)$ and $\mathbf{p}=\left(p_{1}, \ldots, p_{d}\right)$, as the set of functions supported on $\mathscr{D}$, and such that all the constants $C\left(s_{i}, l\right)$ appearing in the definition above are less than $M$.

\subsection{Embeddings and approximation properties for anisotropic Besov spaces.}

2.1.1. Embeddings. As in dimension 1, for the sparse zone, the embeddings are an essential tool. We quote here Theorem 6.9 in [13, p. 252].

Proposition 1. If

$$
\begin{aligned}
& 1 \leqslant p_{i} \leqslant r \leqslant \infty \quad \text { for all } \quad i \in\{1, \ldots, d\}, \\
& K^{r}=\left[1-\sum_{i=1}^{d} \frac{1}{s_{i}}\left(\frac{1}{p_{i}}-\frac{1}{r}\right)\right]>0
\end{aligned}
$$

are satisfied and if we put

$$
\begin{gathered}
K_{j}=\left[1-\sum_{i=1}^{d} \frac{1}{s_{i}}\left(\frac{1}{p_{i}}-\frac{1}{p_{j}}\right)\right], \\
s_{i}^{\prime}=\frac{s_{i} K^{r}}{K_{i}},
\end{gathered}
$$

then the space $B_{\left(p_{1}, \ldots, p_{d}\right), \infty}^{\left(s_{1}, \ldots, s_{d}\right)}$ is embedded in the space $B_{(r, \ldots, r), \infty}^{\left(s_{1}^{\prime}, \ldots, s_{d}^{\prime}\right)}$. 
Notice that for all $i$, because of $p_{i} \leqslant r$, we have $K_{i} \geqslant K^{r}>0$. This proposition will be used in two situations: for $r=p$, then $K^{r}=K^{p}$, and $r=\infty$, where we see the importance of the condition $1-\sum_{i=1}^{d} s_{i}^{-1} p_{i}^{-1}>0$.

2.1.2. Approximation properties. The following proposition is proved in [5], and shows that the approximation properties in the Besov spaces are driven by the regularity parameters $s_{i}$ 's.

Proposition 2. Let $f \in B_{\left(p_{1}, \ldots, p_{d}\right), \infty}^{\left(s_{1}, \ldots, s_{d}\right)}$.

Let $g(t)$ be an integrable function defined on $\mathbf{R}, \int_{\mathbf{R}} g(t) d t=1$. Let

$$
g_{l}(t)=\sum_{k=1}^{l} C_{l}^{k}(-1)^{k+1} k^{-1} g\left(\frac{t}{k}\right) \text {. }
$$

Define

$$
K\left(x_{1}, \ldots, x_{d}\right)=g_{l}\left(x_{1}\right) \cdots g_{l}\left(x_{d}\right) .
$$

For $h$ and $y$ in $\mathbf{R}^{d}$, and $i$ arbitrary in $\{1, \ldots, d\}$, let $[y . h]=\left(y_{1} h_{1}, \ldots, y_{d} h_{d}\right), \quad[y . h]^{i}=\left(y_{1} h_{1}, \ldots, y_{i-1} h_{i-1}, 0, y_{i+1} h_{i+1}, \ldots, y_{d} h_{d}\right)$. Then

$$
\left\|\int_{\mathbf{R}^{d}} K(y)\left[f(x+[y \cdot h])-f\left(x+[y \cdot h]^{i}\right)\right] d y\right\|_{\mathbf{L}_{p_{i}}\left(\mathbf{R}^{d}, d x\right)} \leqslant L\left|h_{i}\right|^{s_{i}} .
$$

2.2. Essential results from [5]. Let us now recall the following ingredients of the proof in [5], which will be useful here.

2.2.1. Dyadic directional modulus of approximation. Let us define $j^{M}(\varepsilon)$ in $\mathbf{N}$ by $2^{-\left(j^{M}(\varepsilon)+1\right)} \leqslant \varepsilon^{2} \leqslant 2^{-j^{M}(\varepsilon)}$, and restrict our attention to the following set of dyadics:

$$
I:=I(\varepsilon)=\left\{j=\left(j_{1}, \ldots, j_{d}\right): 0 \leqslant j_{i} \leqslant j^{M}(\varepsilon) \forall i\right\} .
$$

Define the following dyadic modulus of approximation:

$$
\widetilde{g}_{i}\left(2^{-j_{i}}\right)(t) \triangleq \sup _{y \in \widetilde{D}^{i}\left(2^{-j_{i}}\right)}\left|\int_{\mathbf{R}^{d}} K(x)\left[f(t+[y \cdot x])-f\left(t+[y \cdot x]^{i}\right)\right] d x\right|
$$

where

$$
\begin{aligned}
\widetilde{D}^{i}\left(2^{-j_{i}}\right)=\{ & \left(\delta_{1} 2^{-j_{1}^{\prime}}, \ldots, \delta_{d} 2^{-j_{d}^{\prime}}\right): \\
& \left.\delta_{j} \in\{0,1\}, 0 \leqslant j_{l}^{\prime} \leqslant j^{M}(\varepsilon) \forall l \neq i, j_{i} \leqslant j_{i}^{\prime} \leqslant j^{M}(\varepsilon)\right\} .
\end{aligned}
$$

We shall restrict ourselves to functions having a minimal regularity: For $0<\nu \leqslant 1,0<L_{\nu}<\infty, 0<L<\infty$, we say that the function $f$ belongs to $\mathscr{F}_{0}=\mathscr{F}_{0}\left(\nu, L_{\nu}, L,[0, T]^{d}\right)$ if

(a) $\sup _{t \in[0, T]^{d}}|f(t)| \leqslant L$,

(b) for all $t, t^{\prime} \in[0, T]^{d},\left|f(t)-f\left(t^{\prime}\right)\right| \leqslant L_{\nu}\left(\left|t_{1}-t_{1}^{\prime}\right|^{\nu}+\cdots+\left|t_{d}-t_{d}^{\prime}\right|^{\nu}\right)$.

Notice that Proposition 1 implies that under the condition 1 $\sum_{i=1}^{d} s_{i}^{-1} p_{i}^{-1}>0$, we necessarily consider functions belonging to a set $\mathscr{F}_{0}\left(\nu,[0, T]^{d}\right)$, with $\nu$ eventually small enough but positive. 
2.2.2. «Ideal» multibandwidth. The following proposition is proved in [5] and describes the behavior of the optimal multiscale bandwidth if we restrict the choice to dyadics.

Proposition 3. Consider any arbitrary $f \in \mathscr{F}_{0}$ and $0<\varepsilon<$ $\left(\|K\| /\left(L_{\nu} \int|K(x) \| x|^{\nu} d x\right)\right)^{1 / \nu}$.

1. There exists $\bar{j}=\bar{j}(t)=\left(\bar{j}_{1}, \ldots, \bar{j}_{d}\right) \in I(\varepsilon)$ solution of the following problem:

(a) If $\bar{j}_{i}=0$, then $\tilde{g}_{i}\left(2^{-\bar{j}_{i}}\right)(t) \leqslant \lambda(\bar{j}, \varepsilon)$;

(b) if $j^{M}(\varepsilon) \geqslant \bar{j}_{i}>0$, then $\tilde{g}_{i}\left(2^{-\bar{j}_{i}}\right)(t) \leqslant \lambda(\bar{j}, \varepsilon)$ and $\tilde{g}_{i}\left(2^{-\left(\bar{j}_{i}-1\right)}\right)(t) \geqslant$ $\lambda(\bar{j}-1, \varepsilon)$, where $\bar{j}-1=\left(\bar{j}_{1}-1, \ldots, \bar{j}_{d}-1\right)$.

2. Let $\bar{j}=\left(\bar{j}_{1}, \ldots, \bar{j}_{d}\right)$ and $\bar{j}^{\prime}=\left(\bar{j}_{1}^{\prime}, \ldots, \bar{j}_{d}^{\prime}\right)$ in $I(\varepsilon)$ be two solutions of the previous problem. Then

either $\sum_{k=1}^{d} \bar{j}_{k}^{\prime} \leqslant \sum_{k=1}^{d} \bar{j}_{k} \leqslant \sum_{k=1}^{d} \bar{j}_{k}^{\prime}+d \quad$ or $\quad \sum_{k=1}^{d} \bar{j}_{k} \leqslant \sum_{k=1}^{d} \bar{j}_{k}^{\prime} \leqslant \sum_{k=1}^{d} \bar{j}_{k}+d$.

The last sentence of the proposition shows that if the solution $\bar{j}$ is not unique, then two solutions will satisfy

$$
\sum_{k=1}^{d} \bar{j}_{k}^{\prime}-d \leqslant \sum_{k=1}^{d} \bar{j}_{k} \leqslant \sum_{k=1}^{d} \bar{j}_{k}^{\prime}+d
$$

In the sequel, we will consider $\bar{j}$ a particular solution of the previous proposition, no matter which one it is since all our bounds will only depend on $\sum_{k=1}^{d} j_{k}$.

2.2.3. «Oracle» inequality. Let us recall that

$$
\lambda(j, \varepsilon):=\|K\| 2^{\sum_{i=1}^{d} j_{i} / 2} \Delta, \quad \Delta=\varepsilon\left(1+d \ln \varepsilon^{-1}\right)^{1 / 2} .
$$

Let $f_{\varepsilon}^{*}(\cdot)=\hat{f}_{\varepsilon}\left(\bar{h}_{\varepsilon}(\cdot, \kappa), \cdot\right)$ with $\kappa=(1, \ldots, 1)$ be the estimator constructed in $[5]$.

Applying the pointwise «oracle» inequality proved in [5] (Theorem 1) for this particular choice of the parameter $\kappa$, we arrive at the following result.

Proposition 4. Let $\mathscr{F}$ be included into $\mathscr{F}_{0}\left(\nu, L_{\nu}, L\right)$, then for all $f \in \mathscr{F}$, for any $\varepsilon>0$, and for $t \in[0,1]^{d}$,

$$
\mathbf{E}_{f}\left|f_{\varepsilon}^{*}(t)-f(t)\right|^{p} \leqslant C_{2}(p) \lambda(\bar{j}(t), \varepsilon)^{p} .
$$

The constant $C_{2}(p)$ is explicitly given in [5].

\section{Minimax rates over anisotropic Besov balls}

3.1. Upper bound. Let $f_{\varepsilon}^{*}(\cdot)=\hat{f}_{\varepsilon}\left(\bar{h}_{\varepsilon}(\cdot, \kappa), \cdot\right)$ be the estimator constructed in [5] with $\kappa=(1, \ldots, 1)$.

We have the following theorem. 
Theorem 1. Let $B_{\mathbf{p}, \infty}^{\mathbf{s}}(M)$ be defined as above, with $\mathbf{s}=\left(s_{1}, \ldots, s_{d}\right)$, $\mathbf{p}=\left(p_{1}, \ldots, p_{d}\right) \in \mathbf{R}_{+}^{d}$ and such that

$$
\begin{gathered}
1 \leqslant p_{i} \leqslant p<\infty \quad \text { for all } i \in\{1, \ldots, d\} \\
1-\sum_{i=1}^{d} \frac{1}{s_{i}} \frac{1}{p_{i}}>0
\end{gathered}
$$

Set

$$
\delta=\left[\frac{2}{p}-\sum_{i=1}^{d} \frac{1}{s_{i}}\left(\frac{1}{p_{i}}-\frac{1}{p}\right)\right] .
$$

If $\delta \leqslant 0$ («sparse zone») then, for

$$
L=\left[1-\sum_{i=1}^{d} \frac{1}{s_{i}}\left(\frac{1}{p_{i}}-\frac{1}{2}\right)\right], \quad K=\left[1-\sum_{i=1}^{d} \frac{1}{s_{i}}\left(\frac{1}{p_{i}}-\frac{1}{p}\right)\right]
$$

we have

$$
\begin{aligned}
& \sup _{B_{\mathbf{p}, \infty}^{\mathbf{s}}(M)} \mathbf{E}_{f} \int_{[0,1]^{d}}\left|f_{\varepsilon}^{*}(t)-f(t)\right|^{p} d t \\
& \quad \leqslant C_{5}(p)\left\{\left[\ln \varepsilon^{-1}\right]^{1 / 2} \varepsilon\right\}^{K p / L}\left[\ln \varepsilon^{-1}\right]^{d I\{\delta=0\}},
\end{aligned}
$$

where $C_{5}(p)$ is an absolute constant.

This theorem leads to the following remarks.

1. Let us first note that the behavior of the estimator $f_{\varepsilon}^{*}$ if $\delta>0$ («dense zone») has been investigated in the former paper [5] (Theorem 4). In particular, we deduce from [5] that if the inequalities (5) and (6) of Theorem 1 therein are verified and $\delta>0$, then

$$
\sup _{B_{\mathbf{P}, \infty}^{\mathbf{s}}(M)} \mathbf{E}_{f} \int_{[0,1]^{d}}\left|f_{\varepsilon}^{*}(t)-f(t)\right|^{p} d t \leqslant C_{5}(p)\left\{\left[\ln \varepsilon^{-1}\right]^{1 / 2} \varepsilon\right\}^{2 \bar{s} p /(2 \bar{s}+1)}\left[\ln \varepsilon^{-1}\right]^{d-1}
$$

where $C_{5}(p)$ is an absolute constant, $\bar{s}$ is defined by $1 / \bar{s}=\sum_{i=1}^{d} 1 / s_{i}$.

In [5] this result was treated as adaptive since the estimator does not depend on the parameters of the Besov ball. Actually, the estimator $f_{\varepsilon}^{*}$ is not minimax adaptive if $\delta>0$ : the upper bound given by (16) and the minimax rate of convergence found in [5] (Theorem 3) differ by the factor $\{\ln (1 / \varepsilon)\}^{b}$, where $b=\bar{s} p /(2 \bar{s}+1)+d-1$. In this situation one usually speaks on «adaptation up to a logarithmic factor». However, if $\delta<0$ our estimator $f_{\varepsilon}^{*}$ is minimax adaptive (see remark after Theorem 2).

2. Notice here that when $1-\sum_{i=1}^{d} s_{i}^{-1} p_{i}^{-1}>0$, then $K>0$, hence $\sum_{i=1}^{d} s_{i}^{-1}\left(1 / p_{i}-1 / p\right)<1$, furthermore $\delta \leqslant 0$ implies $\sum_{i=1}^{d} s_{i}^{-1}\left(1 / p_{i}-1 / p\right) \geqslant$ $2 / p$. We deduce that, in the sparse zone, necessarily $p>2$.

As a consequence $L \geqslant K>0$.

3. We observe a phenomenon which is the equivalent in $d$ dimension of the famous «elbow» observed for $d=1$, leading to essentially two regimes 
for the minimax rates of convergence. Notice that for $d=1$, the rate

$$
\frac{K p}{L}=\frac{2\left(s-1 / p_{1}+1 / p\right) p}{2\left(s-1 / p_{1}\right)+1}
$$

is exactly the rate observed in this situation (see [12], [1]).

4. If we notice that the rate can also be denoted by

$$
\frac{K p}{L}=\frac{[\bar{s}-1 / \bar{p}+1 / p] p}{\bar{s}-1 / \bar{p}+\frac{1}{2}} \quad \text { if } \quad \frac{1}{\bar{s}}=\sum_{i=1}^{d} \frac{1}{s_{i}} \quad \text { and } \quad \frac{1}{\bar{p}} \frac{1}{\bar{s}}=\sum_{i=1}^{d} \frac{1}{s_{i}} \frac{1}{p_{i}},
$$

we see that the multidimensional setting can be interpreted as behaving exactly as the one-dimensional case (as recalled above), if we replace $s$ by $\bar{s}$ and $p_{1}$ by $\bar{p}$.

5. As in dimension 1 , the following lemma proves that the rate in the sparse zone is slower than in the dense region.

Lemma 1. Under the conditions of the theorem, if $\delta \leqslant 0$, then

$$
\frac{2 \bar{s}}{1+2 \bar{s}} \geqslant \frac{K}{L}
$$

with equality if and only if $\delta=0$.

The proof of the lemma is just observing that

$$
\frac{K}{L}-\frac{2 \bar{s}}{1+2 \bar{s}}=\frac{\delta}{L(1+2 \bar{s})}
$$

and, obviously,

$$
L=K+\sum_{j=1}^{d} s_{j}^{-1}\left(\frac{1}{2}-\frac{1}{p}\right)>0 .
$$

6. We can also observe in the following lemma, that, for the sparse case, as well as the dense case, the rates are strictly decreasing with the dimension.

Lemma 2. Under the conditions of the theorem, if $\left\{i_{1}, \ldots, i_{l}\right\} \subset$ $\left\{i_{1}, \ldots, i_{m}\right\}$, then

$$
\bar{s}\left(i_{1}, \ldots, i_{m}\right) \leqslant \bar{s}\left(i_{1}, \ldots, i_{l}\right)
$$

and

$$
\frac{K\left(i_{1}, \ldots, i_{l}\right)}{L\left(i_{1}, \ldots, i_{l}\right)} \geqslant \frac{K\left(i_{1}, \ldots, i_{m}\right)}{L\left(i_{1}, \ldots, i_{m}\right)}
$$

where $\bar{s}\left(i_{1}, \ldots, i_{m}\right)^{-1}=\sum_{r=1}^{l} \frac{1}{s_{i_{r}}}$ and

$$
\begin{aligned}
& K\left(i_{1}, \ldots, i_{l}\right)=\left[1-\sum_{r=1}^{l} \frac{1}{s_{i_{r}}}\left(\frac{1}{p_{i_{r}}}-\frac{1}{p}\right)\right], \\
& L\left(i_{1}, \ldots, i_{l}\right)=\left[1-\sum_{i=1}^{l} \frac{1}{s_{i_{r}}}\left(\frac{1}{p_{i_{r}}}-\frac{1}{2}\right)\right],
\end{aligned}
$$

and equalities in (18) or (19) only occur if the two sets are equal. 
P r o o f. (18) is obvious. (19) is obtained by induction: Using the symmetry of the problem, we only need to calculate

$$
\frac{K(1, \ldots, d)}{L(1, \ldots, d)}-\frac{K(1, \ldots, d-1)}{L(1, \ldots, d-1)}=\frac{\left(\frac{1}{2}-1 / p\right)\left[1-\sum_{i=1}^{d} s_{i}^{-1}\left(1 / p_{i}-1 / p_{d}\right)\right]}{L(1, \ldots, d) L(1, \ldots, d-1)}
$$

Now, since $p_{i} \leqslant p, K>0$ implies that $\left[1-\sum_{i=1}^{d} s_{i}^{-1}\left(1 / p_{i}-1 / p_{d}\right)\right] \geqslant K>0$, as well as $K\left(i_{1}, \ldots, i_{l}\right)>0$, for any $l \geqslant 1$.

Moreover, obviously,

$$
L\left(i_{1}, \ldots, i_{l}\right)=K\left(i_{1}, \ldots, i_{l}\right)+\sum_{j=1}^{l} \frac{1}{s_{i_{j}}}\left(\frac{1}{2}-\frac{1}{p}\right)>0 .
$$

3.2. Lower bound. In this section, we state the following lower bound result.

Theorem 2. Let $L$ and $K$ be the constants defined in Theorem 1 . Then for $\varepsilon>0$ small enough,

$$
\inf _{\tilde{f}} \sup _{f \in B_{\mathbf{p}, \infty}^{\mathbf{s}}(M)} \mathbf{E}_{f} \int_{[0,1]^{d}}\left|\tilde{f}_{\varepsilon}(t)-f(t)\right|^{p} d t \geqslant C_{6}(p)\left\{\left[\ln \varepsilon^{-1}\right]^{1 / 2} \varepsilon\right\}^{K p / L},
$$

where $C_{6}(p)$ is an absolute constant and the infimum is taken over all possible estimators.

$\mathrm{R}$ e $\mathrm{m}$ a $\mathrm{rk}$. Let us note that this lower bound is valid for a standard Besov ball without any restriction on its parameters. However, the bound is effective only for $\delta<0$. For $\delta=0$, the upper and lower bounds differ by a logarithmic factor. As is obvious, this lower bound is correct but not sharp for the case $\delta>0$ («dense zone») and, in particular, another proof has to be used, see [5].

$\mathrm{R}$ e $\mathrm{m}$ a r k s. 1. We deduce from Theorems 1 and 2 that the estimator $f_{\varepsilon}^{*}$ is minimax adaptive in the sparse zone $\delta<0$.

2. We have from Theorem 1 , Theorem 2, and inequality (16) that our estimator is adaptive up to a logarithmic factor with respect to the family of Besov balls $B_{\mathbf{p}, \infty}^{\mathbf{s}}(M)$ with $\mathbf{s}$ and p satisfying (12) and (13).

3. It is an open question how to construct an estimator being minimax adaptive with respect to the family of Besov balls $B_{\mathbf{p}, \infty}^{\mathbf{s}}(M)$ with $\mathbf{s}$ and $\mathbf{p}$ satisfying (12) and (13).

\section{Proof of Theorem 1}

The proof of inequality (15) heavily builds as above on the following decomposition. Using (12), we get

$$
\begin{aligned}
& \mathbf{E}_{f} \int\left|f_{\varepsilon}^{*}(t)-f(t)\right|^{p} d t \\
& \quad \leqslant C_{2}(p) \sum_{j=\left(j_{1}, \ldots, j_{d}\right) \in I} \lambda(j, \varepsilon)^{p} \mu\left\{\bar{j}_{i}=j_{i} \forall i \in\{1, \ldots, d\}\right\} .
\end{aligned}
$$


Lemma 3. For $f \in B_{q_{i}, \infty}^{t_{i}},\left(j_{1}, \ldots, j_{d}\right) \in \mathbf{N}$, if $j_{i} \geqslant 1$, then

$$
\mu\left\{\bar{j}_{i}=j_{i} \forall i \in\{1, \ldots, d\}\right\} \leqslant \lambda(j, \varepsilon)^{-q_{i}} 2^{-j_{i} t_{i} q_{i}} .
$$

$\mathrm{Pr}$ o of of $\mathrm{L} \mathrm{e} \mathrm{m} \mathrm{ma} \mathrm{3.} \mathrm{Using} \mathrm{Propositions} 3$ and 2, we have for $j_{i} \geqslant 1$

$$
\begin{aligned}
& \mu\left\{\bar{j}_{i}=j_{i} \forall i \in\{1, \ldots, d\}\right\} \leqslant \mu\left\{t \in[0,1]: \widetilde{g}_{i}\left(2^{-\left(j_{i}-1\right)}\right)(t) \geqslant \lambda(j, \varepsilon)\right\} \\
& \leqslant \lambda(j, \varepsilon)^{-q_{i}}\left\|\tilde{g}_{i}\left(2^{-\left(j_{i}-1\right)}\right)(\cdot)\right\|_{\mathbf{L}_{\boldsymbol{q}_{i}}(d t)}^{q_{i^{2}}} \leqslant \lambda(j, \varepsilon)^{-q_{i}} 2^{-j_{i} t_{i} \boldsymbol{q}_{i}} .
\end{aligned}
$$

As can be observed in the preceding lemma, we have to distinguish between the cases $j_{i}=0$ and $j_{i} \geqslant 1$. Hence the bound in (21) can be rewritten as

$$
\begin{aligned}
\sum_{m=0}^{d} \sum_{j_{i_{1}}, \ldots, j_{i_{m}} \in\left\{1, \ldots, j^{M}(\varepsilon)\right\}} \lambda(j, \varepsilon)^{p} \mu\left\{\bar{j}_{i_{l}}\right. & =j_{i_{l}} \forall l \in\{1, \ldots, m\}, \\
\bar{j}_{i_{l}} & =0 \quad \forall l \notin\{1, \ldots, m\}\} .
\end{aligned}
$$

First, let us observe that the term corresponding to $m=0$ is bounded by $\Delta^{p / 2}$ and is not significant compared to the rate announced in the theorem.

To simplify the notation, we shall omit in the sequel the indication $\bar{j}_{i_{l}}=0$ for all $l \notin\{1, \ldots, m\}$ and introduce the quantities

$$
r_{i}=\frac{p-p_{i}}{2}, \quad q_{i}=p_{i} s_{i}, \quad i \in\{1, \ldots, d\}
$$

in such a way that $\delta=2 p^{-1}\left(1-\sum_{i=1}^{d} r_{i} / q_{i}\right)$, so

$$
\delta \leqslant 0 \Longleftrightarrow \sum_{i=1}^{d} \frac{r_{i}}{q_{i}} \geqslant 1 .
$$

Now, let us observe that because we are going to consider only a part of the $d$ dimensions, we have to distinguish between the two following cases:

1. $\left(i_{1}, \ldots, i_{m}\right) \in \mathscr{C}_{+}=\left\{\left(i_{1}, \ldots, i_{m}\right): \sum_{l=1}^{m} r_{i_{l}} / q_{i_{l}}<1\right\}$,

2. $\left(i_{1}, \ldots, i_{m}\right) \in \mathscr{C}_{-}=\left\{\left(i_{1}, \ldots, i_{m}\right): \sum_{l=1}^{m} r_{i_{l}} / q_{i_{l}} \geqslant 1\right\}$.

In such a way (22) (omitting the term corresponding to $m=0$ ) may be replaced by

$$
\begin{aligned}
\sum_{m=1}^{d} & {\left[\sum_{\left(i_{1}, \ldots, i_{m}\right) \in \mathscr{C}_{+}} \sum_{j_{i_{1}}, \ldots, j_{i_{m}} \in\left\{1, \ldots, j^{M}(\varepsilon)\right\}} \lambda(j, \varepsilon)^{p} \mu\left\{\bar{j}_{i_{l}}=j_{i_{l}} \forall l \in\{1, \ldots, m\}\right\}\right.} \\
& \left.+\sum_{\left(i_{1}, \ldots, i_{m}\right) \in \mathscr{C}_{-}} \sum_{j_{i_{1}}, \ldots, j_{i_{m}} \in\left\{1, \ldots, j^{M}(\varepsilon)\right\}} \lambda(j, \varepsilon)^{p} \mu\left\{\bar{j}_{i_{l}}=j_{i_{l}} \quad \forall l \in\{1, \ldots, m\}\right\}\right] .
\end{aligned}
$$


4.1. Bound for the $\mathscr{C}_{+}$-terms. Using (21) we get

$$
\begin{aligned}
& \sum_{m=1}^{d} \sum_{\left(i_{1}, \ldots, i_{m}\right) \in \mathscr{C}_{+} j_{i_{1}}, \ldots, j_{i_{m}} \in\left\{1, \ldots, j^{M}(\varepsilon)\right\}} \lambda(j, \varepsilon)^{p} \mu\left\{\bar{j}_{i_{l}}=j_{i_{l}} \forall l \in\{1, \ldots, m\}\right\} \\
& \leqslant \sum_{m=1}^{d} \sum_{\left(i_{1}, \ldots, i_{m}\right) \in \mathscr{C}_{+}} C_{5}(p) \Delta^{2 p \bar{s}\left(i_{1}, \ldots, i_{m}\right) /\left(1+2 \bar{s}\left(i_{1}, \ldots, i_{m}\right)\right)}\left(\ln \varepsilon^{-1}\right)^{m-1},
\end{aligned}
$$

where $1 / \bar{s}\left(i_{1}, \ldots, i_{m}\right)=\sum_{l=1}^{m} 1 / s_{i_{l}}$.

Using Lemmas 1 and 2 , we get

$$
\begin{aligned}
& \sum_{m=1}^{d} \sum_{\left(i_{1}, \ldots, i_{m}\right) \in \mathscr{C}_{+}} C_{5}(p) \Delta^{2 p \bar{s}\left(i_{1}, \ldots, i_{m}\right) /\left(1+2 \bar{s}\left(i_{1}, \ldots, i_{m}\right)\right)}\left(\ln \varepsilon^{-1}\right)^{m-1} \\
& \quad \leqslant \sum_{m=1}^{d} \sum_{\left(i_{1}, \ldots, i_{m}\right) \in \mathscr{C}_{+}} C_{5}(p) \Delta^{2 p \bar{s} /(1+2 \bar{s})}\left(\ln \varepsilon^{-1}\right)^{d-1} \\
& \leqslant 2^{d} C_{5}(p) \Delta^{p K / L}\left(\ln \varepsilon^{-1}\right)^{d I\{\delta=0\}} .
\end{aligned}
$$

4.2. Bound for the $\mathscr{C}_{-}$-terms. Let us now concentrate on the indices lying in $\mathscr{C}_{-}$. Using again Lemma 2 , we only need, as above, to prove the result for the full set of indices.

Now, we will establish the following lemma.

\section{Lemma 4.}

$$
\begin{gathered}
\sum_{j_{1}, \ldots, j_{d} \in\left\{1, \ldots, j^{M}(\varepsilon)\right\}} \lambda(j, \varepsilon)^{p} \mu\left\{\bar{j}_{l}=j_{l} \forall l \in\{1, \ldots, d\}\right\} \\
\leqslant \sum_{j_{1}, \ldots, j_{d} \in\left\{1, \ldots, j^{M}(\varepsilon)\right\}} \min \left\{\left[\lambda(j, \varepsilon)^{p-p_{i}} 2^{-j_{i} s_{i} p_{i}} \wedge 2^{-j_{i} s_{i}^{\prime} p_{i}}\right],\right. \\
i=1, \ldots, d\},
\end{gathered}
$$

where the $s_{i}^{\prime}$ 's are given in Proposition 1, see (9).

This lemma is a consequence of Lemma 3 , which is applied once with $q=p_{i}, t_{i}=s_{i}$, and the second time with $q=p, t_{i}=s_{i}^{\prime}$ using Proposition 1.

Now, let us introduce the following quantities:

$$
\begin{aligned}
& Z_{i}=[\|K\| \Delta]^{K_{i} /\left(L s_{i}\right)} \quad \text { for all } i \in\{1, \ldots, d\}, \\
& 2^{-j_{i}^{*}} \leqslant Z_{i} \leqslant 2^{-j_{i}^{*}+1} \quad \text { for all } i \in\{1, \ldots, d\} .
\end{aligned}
$$

We have the following lemma.

Lemma 5. The following assertions are true at least for $\varepsilon$ small enough:

$$
\begin{aligned}
& 0 \leqslant j_{i}^{*} \leqslant j^{M}(\varepsilon) \quad \text { for all } i \in\{1, \ldots, d\}, \\
& \begin{array}{l}
(\|K\| \Delta)^{p-p_{i}} \prod_{i=1}^{d} Z_{i}^{\left(p_{i}-p\right) / 2} Z_{i}^{p_{i} s_{i}} \\
=Z_{i}^{p s_{i}^{\prime}}=(\|K\| \Delta)^{K p / L} \quad \text { for all } i \in\{1, \ldots, d\} .
\end{array}
\end{aligned}
$$


Note that

(i) (30) is a simple calculation involving the definitions of $K, L, K_{i}, s_{i}^{\prime}$ and using the following identity:

$$
\sum_{i=1}^{d} \frac{K_{i}}{s_{i}}=\frac{1}{\bar{s}}
$$

(ii) (29) follows from $K_{i} /\left(L s_{i}\right) \geqslant 0$ and the fact that (using (31)) $K_{i} / s_{i} \leqslant$ $\sum_{i=1}^{d} K_{i} / s_{i}=1 /(L \bar{s})<2$ since $L \bar{s}=\frac{1}{2}+\bar{s}\left(1-\sum_{i=1}^{d} 1 /\left(s_{i} p_{i}\right)>\frac{1}{2}\right.$.

Hence, putting together Lemma 4 and Lemma 5, we get

$$
\begin{aligned}
& \sum_{j_{1}, \ldots, j_{d} \in\left\{1, \ldots, j^{M}(\varepsilon)\right\}} \lambda(j, \varepsilon)^{p} \mu\left\{\bar{j}_{l}=j_{l} \forall l \in\{1, \ldots, d\}\right\} \\
\leqslant & (\|K\| \Delta)^{K p / L} \\
& \times \sum_{j_{1}, \ldots, j_{d} \in\left\{1, \ldots, j^{M}(\varepsilon)\right\}} \min _{i=1, \ldots, d}\left[2^{\sum_{l=1}^{d}\left(j_{l}-j_{l}^{*}\right)\left(p-p_{i}\right) / 2} 2^{-\left(j_{i}-j_{i}^{*}\right) s_{i} p_{i}} \wedge 2^{-\left(j_{i}-j_{i}^{*}\right) s_{i}^{\prime} p_{i}}\right] \\
\leqslant & (\|K\| \Delta)^{K p / L} \sum_{i=1, \ldots, d}\left[2^{\sum_{l=1}^{d} j_{l}\left(p-p_{i}\right) / 2} 2^{-j_{i} s_{i} p_{i}} \wedge 2^{-j_{i} s_{i}^{\prime} p_{i}}\right] .
\end{aligned}
$$

4.3. Barycentering. Thus we obtain a sum where all possible configurations of signs appear. We will investigate a standard configuration. To simplify, let us put $\lambda_{i}=s_{i}^{\prime} p$ and recall that $r_{i}=\left(p-p_{i}\right) / 2, q_{i}=p_{i} s_{i}$. Then a standard configuration of the previous sum can be written and bounded in the following way:

$$
\begin{aligned}
& \sum_{j_{1}, \ldots, j_{s} \in\left\{0, \ldots, j^{M}(\varepsilon)\right\}} \sum_{j_{s+1}, \ldots, j_{d} \in\left\{-j^{M}(\varepsilon), \ldots, 0\right\}} \min _{i=1, \ldots, p}\left[2^{\sum_{l=1}^{d} j_{l} r_{i}} 2^{-j_{i} q_{i}} \wedge 2^{-j_{i} \lambda_{i}}\right] \\
\leqslant & \sum_{j_{1}, \ldots, j_{s} \in\left\{0, \ldots, j^{M}(\varepsilon)\right\}} \sum_{j_{s+1}, \ldots, j_{d} \in\left\{-j^{M}(\varepsilon), \ldots, 0\right\}} \prod_{i=1}^{d} 2^{-j_{i} \lambda_{i} \alpha_{i}} \prod_{i=1}^{d} 2^{\left(-j_{i} q_{i}+r_{i} \sum_{l=1}^{d} j_{l}\right) \beta_{i}}
\end{aligned}
$$

for a collection of nonnegative real $\alpha_{i}, \beta_{i}$ such that $\sum_{i=1}^{d}\left(\alpha_{i}+\beta_{i}\right)=1$.

Let us choose

$$
\alpha_{s+1}=\cdots=\alpha_{d}=0, \quad \beta_{i}=\frac{1}{R q_{i}}, \quad i \in\{1, \ldots, d\}
$$

$R$ and $\alpha_{1}, \ldots, \alpha_{s}$ will be chosen later. If we denote by $\mathscr{R}$ the set of indices considered above: $\left\{j_{1}, \ldots, j_{s} \in\left\{0, \ldots, j^{M}(\varepsilon)\right\}, j_{s+1}, \ldots, j_{d} \in\right.$ $\left.\left\{-j^{M}(\varepsilon), \ldots, 0\right\}\right\}$, then we get using (33):

$$
\begin{aligned}
& \sum_{\mathscr{R}} \min \left\{2^{\sum_{l=1}^{d} j_{l} r_{i}} 2^{-j_{i} q_{i}} \wedge 2^{-j_{i} \lambda_{i}}, i=1, \ldots, d\right\} \\
& \quad \leqslant \sum_{\mathscr{R}} \prod_{i \leqslant s} 2^{-j_{i}\left(\lambda_{i} \alpha_{i}+\left[1-\sum_{i=1}^{d} r_{i} / q_{i}\right] / R\right)} \prod_{i \geqslant s+1} 2^{-j_{i}\left(\left[1-\sum_{i=1}^{d} r_{i} / q_{i}\right] / R\right)} .
\end{aligned}
$$


As we observed

$$
\delta<0 \Longleftrightarrow \sum_{i=1}^{d} \frac{r_{i}}{q_{i}}>1 .
$$

Hence, in this case, the last $d-s$ terms are affected with a positive power, whereas if we choose $\alpha_{i}=\left[u_{i}-1+\sum_{i=1}^{d} r_{i} / q_{i}\right] /\left(R \lambda_{i}\right)$ with $u_{i}>0$, then the first $s$ terms are affected with a negative power, in such a way that the sum is convergent. It remains to show that this combination can be done together with the constraint $\sum_{i=1}^{d}\left(\alpha_{i}+\beta_{i}\right)=1$. But this is equivalent to

$$
\frac{1}{R}\left[\left(u_{i}-1+\sum_{i=1}^{d} \frac{r_{i}}{q_{i}}\right) \frac{1}{\lambda_{i}}+\sum_{i=1}^{d} \frac{1}{q_{i}}\right]=1
$$

which is always possible by chosing $R$ in an appropriate way.

It remains to see that in the case $\delta=0$ we have $\sum_{i=1}^{d} r_{i} / q_{i}=1$, and the sum may only be bounded by $\left(\ln \varepsilon^{-1}\right)^{d-s}$.

\section{Proof of Theorem 2}

5.1. Some general results. The proofs of the results presented in this section are absolutely standard and for this reason are given with a short proof.

5.1.1. Lower bound for an abstract model. Let $\left(\Omega^{\varepsilon}, V^{\varepsilon}, \mathbf{P}_{f}^{\varepsilon}\right.$, $f \in \mathscr{F}$ ) be a sequence of statistical experiments generated by the observation $X^{(\varepsilon)}$ and let $G: \mathscr{F} \rightarrow \Lambda$ be the functional to be estimated. Here $\Lambda$ is some normed vector space and let $\|\cdot\|$ be the corresponding norm. Let us suppose that the following assumptions are fulfilled.

For any $\varepsilon>0$ there exist an integer $N_{\varepsilon}$ and a set of parameters $f_{i}=f_{i}^{(\varepsilon)}$, $i=0,1, \ldots, N_{\varepsilon}$, such that

a. $\left\{f_{i}, i=0,1, \ldots, N_{\varepsilon}\right\} \subset \mathscr{F}$;

b. $\left\|G\left(f_{i}\right)-G\left(f_{0}\right)\right\|=a_{\varepsilon}$ for all $i=1, \ldots, N_{\varepsilon}$;

c. $\liminf \inf _{\varepsilon \rightarrow 0} \sup _{\tau \in[0,1]} \mathbf{E}_{f_{0}}^{\varepsilon} \min \left[\tau Z_{\varepsilon}, 1-\tau\right] \triangleq K>0$.

Here $\mathbf{E}_{f}^{\varepsilon}$ is the expectation with respect to probability measure $\mathbf{P}_{f}^{\varepsilon}$ and

$$
Z_{\varepsilon}=\frac{1}{N_{\varepsilon}} \sum_{i=1}^{N_{\varepsilon}} z_{i}\left(X^{(\varepsilon)}\right), \quad z_{i}\left(X^{(\varepsilon)}\right)=\frac{d \mathbf{P}_{f_{i}}^{\varepsilon}}{d \mathbf{P}_{f_{0}}^{\varepsilon}}\left(X^{(\varepsilon)}\right) .
$$

Proposition 5. Suppose that the assumptions $\mathbf{a}, \mathbf{b}$, and $\mathbf{c}$ are fulfilled, then for all $q>0$

$$
\liminf _{\varepsilon \rightarrow 0} \inf _{\widetilde{G}} \sup _{f \in \mathscr{F}} \mathbf{E}_{f}^{\varepsilon}\left(a_{\varepsilon}^{-1}\|\widetilde{G}-G(f)\|\right)^{q} \geqslant K \min \left(2^{1-q}, 1\right)
$$

where the infimum is taken over all measurable functions (with respect to $\left.X^{(\varepsilon)}\right)$ and with values in $\Lambda$. 
Proof of Proposition 5 . Set for arbitrary $\widetilde{G}$

$$
R_{\varepsilon}(\widetilde{G})=\sup _{f \in \mathscr{F}} \mathbf{E}_{f}^{\varepsilon}\left(a_{\varepsilon}^{-1}\|\widetilde{G}-G(f)\|\right)^{q}, \quad T=T_{\varepsilon}=a_{\varepsilon}^{-1}\left\|\widetilde{G}-G\left(f_{0}\right)\right\| .
$$

Using the triangular inequality and assumptions $\mathbf{a}, \mathbf{b}$ we have for all $\tau \in[0,1]$

$$
\begin{aligned}
R_{\varepsilon}(\widetilde{G}) \geqslant & (1-\tau) \mathbf{E}_{f_{0}}^{\varepsilon}\left(a_{\varepsilon}^{-1}\left\|\widetilde{G}-G\left(f_{0}\right)\right\|\right)^{q} \\
& +\frac{\tau}{N_{\varepsilon}} \sum_{i=1}^{N_{\varepsilon}} \mathbf{E}_{f_{i}}^{\varepsilon}\left(a_{\varepsilon}^{-1}\left|\left\|\widetilde{G}-G\left(f_{0}\right)\right\|-\left\|G\left(f_{i}\right)-G\left(f_{0}\right)\right\|\right|\right)^{q} \\
= & (1-\tau) \mathbf{E}_{f_{0}}^{\varepsilon}(T)^{q}+\frac{\tau}{N_{\varepsilon}} \sum_{i=1}^{N_{\varepsilon}} \mathbf{E}_{f_{i}}^{\varepsilon}|T-1|^{q} \\
= & \mathbf{E}_{f_{0}}^{\varepsilon}\left[(1-\tau)(T)^{q}+\tau Z_{\varepsilon}|T-1|^{q}\right] \\
\geqslant & \mathbf{E}_{f_{0}}^{\varepsilon}\left\{\left[(T)^{q}+|T-1|^{q}\right] \min \left[\tau Z_{\varepsilon}, 1-\tau\right]\right\} \\
\geqslant & \left(\inf _{x \geqslant 0}\left[x^{q}+|x-1|^{q}\right]\right) \mathbf{E}_{f_{0}}^{\varepsilon} \min \left[\tau Z_{\varepsilon}, 1-\tau\right] \\
\geqslant & \left(\min \left[2^{1-q}, 1\right]\right) \mathbf{E}_{f_{0}}^{\varepsilon} \min \left[\tau Z_{\varepsilon}, 1-\tau\right] .
\end{aligned}
$$

The right-hand side of the last inequality does not depend on $\widetilde{G}, \tau$ is an arbitrary real number in $[0,1]$, and applying assumption $\mathbf{c}$ we arrive at the statement of the proposition.

$\mathrm{R} \mathrm{e} \mathrm{m}$ a r k. The same proof remains valid for $q=0$ if one understands $T^{0}$ as $I_{\{A\}}$ and $|T-1|^{0}$ as $I_{\left\{A^{(c)}\right\}}$, where $A$ is a random event belonging to the $\sigma$-algebra generated by the observation $X^{(\varepsilon)}$. This type of risks corresponds to the hypothesis testing problem.

Let us now briefly discuss how to check the assumption $\mathbf{c}$.

Corollary 1. Suppose that the following condition is fulfilled.

$\mathbf{c}^{\prime} . Z_{\varepsilon} \rightarrow 1$ in $\mathbf{P}_{f_{0}}^{\varepsilon}$-probability as $\varepsilon \rightarrow 0$.

Then the assumption $\mathbf{c}$ is verified and $K=\frac{1}{2}$.

This statement is obvious. The optimal choice of the parameter $\tau$ is $\tau=\frac{1}{2}$.

Corollary 2. Suppose that the following condition is fulfilled.

$\mathbf{c}^{\prime \prime} . \lim \sup _{\varepsilon \rightarrow 0} \mathbf{E}_{f_{0}}^{\varepsilon}\left(Z_{\varepsilon}-1\right)^{2} \triangleq \Omega<\infty$.

Then the assumption $\mathbf{c}$ is verified and $K \geqslant \frac{1}{2}(1-\sqrt{\Omega /(\Omega+4)})$.

$\mathrm{R}$ e $\mathrm{m}$ a $\mathrm{r}$ k. Note that $\Omega=0$ implies the assumption $\mathbf{c}^{\prime}$ and the bounds given by Corollary 1 and Corollary 2 coincide.

Proof of Corollary 2 . Note that $\min \left[\tau Z_{\varepsilon}, 1-\tau\right]=\frac{1}{2}\left(\tau Z_{\varepsilon}+\right.$ $\left.1-\tau-\left|\tau Z_{\varepsilon}-(1-\tau)\right|\right)$, therefore

$$
\mathbf{E}_{f_{0}}^{\varepsilon} \min \left[\tau Z_{\varepsilon}, 1-\tau\right]=\frac{1}{2}\left(1-\mathbf{E}_{f_{0}}^{\varepsilon}\left|\tau\left(Z_{\varepsilon}-1\right)-(1-2 \tau)\right|\right) .
$$

Set $\Omega_{\varepsilon}=\mathbf{E}_{f_{0}}^{\varepsilon}\left(Z_{\varepsilon}-1\right)^{2}$. Obviously,

$$
\mathbf{E}_{f_{0}}^{\varepsilon}\left|\tau\left(Z_{\varepsilon}-1\right)-(1-2 \tau)\right| \leqslant \sqrt{\tau^{2} \Omega_{\varepsilon}+(2 \tau-1)^{2}} .
$$


Minimizing the right-hand side of this inequality with respect to $\tau \in[0,1]$ and letting $\varepsilon$ tend to zero, we arrive at the statement of the corollary.

Let us now return to the Gaussian White Noise (GWN) model.

5.1.2. General lower bound for GWN model. It is remarkable that for the GWN model we have a simple explicit condition allowing us to check the assumption $\mathbf{c}^{\prime \prime}$ and, therefore, in view of Corollary 2 to check the assumption c. Also, in this section we will assume that $N_{\varepsilon} \rightarrow \infty$ as $\varepsilon \rightarrow 0$. It is worthwhile to mention that all the results remain valid without this condition and that only some constants might be changed. Moreover, the case $N_{\varepsilon} \rightarrow \infty$ as $\varepsilon \rightarrow 0$ is the most important one.

$\mathrm{R}$ e $\mathrm{m}$ a r k. Remind that the definition of the GWN model requires to suppose that $\mathscr{F} \subset L_{2}(D), D \subseteq \mathbf{R}^{d}$.

Proposition 6. Let $\left(\Omega^{\varepsilon}, V^{\varepsilon}, \mathbf{P}_{f}^{\varepsilon}, f \in \mathscr{F} \subset L_{2}(D)\right)$ be the $G W N$ model.

I. $\Omega=\limsup _{\varepsilon \rightarrow 0} N_{\varepsilon}^{-2} \sum_{i, j=1}^{N_{\varepsilon}} \exp \left\{\varepsilon^{-2}\left\langle f_{i}-f_{0}, f_{j}-f_{0}\right\rangle\right\}-1$.

II. Suppose that there exist $f_{i} \in \mathscr{F}, i=0,1, \ldots, N_{\varepsilon}$, such that the following conditions are verified.

$\mathbf{c}_{1} . \sup _{\left\{i, j=1, \ldots, N_{\varepsilon}, i \neq j\right\}}\left\langle f_{i}-f_{0}, f_{j}-f_{0}\right\rangle \leqslant \mathfrak{M} \varepsilon^{2}$, where $\mathfrak{M}$ is a constant independent of $\varepsilon$ and $\langle\cdot, \cdot\rangle$ is inner product.

$\mathbf{c}_{2} . \sup _{i=1, \ldots, N_{\varepsilon}}\left\|f_{i}-f_{0}\right\|_{2} \leqslant \varepsilon \sqrt{\rho \ln N_{\varepsilon}}$, where $0<\rho<1$ is independent on $\varepsilon$ and $\|\cdot\|_{2}$ is $L_{2}$-norm.

Then the assumption $\mathbf{c}^{\prime \prime}$ is fulfilled and $\Omega \leqslant e^{\mathfrak{M}}-1$.

$\mathrm{R}$ e m a r ks. If $\mathfrak{M}=0$, then $\mathscr{M}=0$ and one can use the lower bound given by Corollary 1 .

If $N_{\varepsilon} \nrightarrow \infty$ as $\varepsilon \rightarrow 0$, then $\mathscr{M} \leqslant e^{\mathfrak{M}}$.

Thus, combining the statements of Proposition 5, Corollary 2, and Proposition 6 we arrive at the following result for the GWN model.

Proposition 7. Let $\left(\Omega^{\varepsilon}, V^{\varepsilon}, \mathbf{P}_{f}^{\varepsilon}, f \in \mathscr{F}\right)$ be the $G W N$ model. Suppose that the following assumptions are fulfilled. For all $\varepsilon>0$ there exist an integer $N_{\varepsilon}, N_{\varepsilon} \rightarrow \infty, \varepsilon \rightarrow 0$, and $f_{i}=f_{i}^{(\varepsilon)} \in L_{2}(D), i=0,1, \ldots, N_{\varepsilon}$, such that

a. $\left\{f_{i}, i=0,1, \ldots, N_{\varepsilon}\right\} \subset \mathscr{F}$

b. $\left\|G\left(f_{i}\right)-G\left(f_{0}\right)\right\|=a_{\varepsilon}$ for all $i=1, \ldots, N_{\varepsilon}$;

$\mathbf{c}_{1} \cdot \sup _{\left\{i, j=1, \ldots, N_{\varepsilon}, i \neq j\right\}}\left\langle f_{i}-f_{0}, f_{j}-f_{0}\right\rangle \leqslant \mathfrak{M} \varepsilon^{2}$, where $\mathfrak{M}$ is an absolute constant;

$\mathbf{c}_{2} . \sup _{i=1, \ldots, N_{\varepsilon}}\left\|f_{i}-f_{0}\right\|_{2} \leqslant \varepsilon \sqrt{\rho \ln N_{\varepsilon}}$ with $0<\rho<1$, then for any $q>0$

$$
\liminf _{\varepsilon \rightarrow 0} \inf _{\widetilde{G}} \sup _{f \in \mathscr{F}^{\varepsilon}} \mathbf{E}_{f}^{\varepsilon}\left(a_{\varepsilon}^{-1}\|\widetilde{G}-G(f)\|\right)^{q} \geqslant\left(1-\sqrt{\frac{e^{\mathfrak{M}}-1}{e^{\mathfrak{M}}+3}}\right) \min \left(2^{-q}, 2^{-1}\right),
$$

where the infimum is taken over all measurable functions (with respect to $\left.X^{(\varepsilon)}\right)$ with values in $\Lambda$. 
$\mathrm{R}$ e m a $\mathrm{rk}$. There exist examples, where the assumptions $\mathbf{c}_{1}$ and $\mathbf{c}_{2}$ are not verified but $\mathscr{M}$ is still finite. In such situations one has to calculate directly the expression given by $\mathbf{I}$ in Proposition 6 .

Proof of Proposition 6. Since we deal with the GWN model, for all $i=1, \ldots, N_{\varepsilon}$,

$$
\begin{aligned}
z_{i}\left(X^{(\varepsilon)}\right) & =\exp \left\{\frac{1}{\varepsilon^{2}} \int_{D}\left(f_{i}-f_{0}\right) X_{\varepsilon}(d t)-\frac{1}{2 \varepsilon^{2}}\left[\left\|f_{i}\right\|_{2}^{2}-\left\|f_{0}\right\|_{2}^{2}\right]\right\} \\
& =\exp \left\{\frac{1}{\varepsilon^{2}} \int_{D}\left(f_{i}-f_{0}\right)\left(X_{\varepsilon}(d t)-f_{0} d t\right)-\frac{1}{2 \varepsilon^{2}}\left\|f_{i}-f_{0}\right\|_{2}^{2}\right\},
\end{aligned}
$$

where for any function $g \in L_{2}(D)$

$$
\int_{D}\left(g-f_{0}\right)\left(X_{\varepsilon}(d t)-f_{0} d t\right) \sim \mathscr{N}\left(0, \varepsilon^{2}\left\|g-f_{0}\right\|_{2}^{2}\right)
$$

with respect to $\mathbf{P}_{f_{0}}^{\varepsilon}$-probability. Remind also that $\mathbf{E}_{f_{0}}^{\varepsilon} z_{i}\left(X^{(\varepsilon)}\right)=1$ for all $i=1, \ldots, N_{\varepsilon}$.

Thus, we find that

$$
\mathbf{E}_{f_{0}}^{\varepsilon}\left(Z_{\varepsilon}-1\right)^{2}=\frac{1}{N_{\varepsilon}^{2}} \sum_{i, j=1}^{N_{\varepsilon}} \exp \left\{\frac{1}{\varepsilon^{2}}\left\langle f_{i}-f_{0}, f_{j}-f_{0}\right\rangle\right\}-1
$$

and, therefore, $\mathbf{I}$ is proved.

Using the assumptions $\mathbf{c}_{1}$ and $\mathbf{c}_{2}$, we obtain

$$
\mathbf{E}_{f_{0}}^{\varepsilon}\left(Z_{\varepsilon}-1\right)^{2} \leqslant N_{\varepsilon}^{\rho-1}+\frac{N_{\varepsilon}-1}{N_{\varepsilon}} e^{\mathfrak{M}}-1 .
$$

Taking into account that $\rho<1$ and $N_{\varepsilon} \rightarrow \infty$ as $\varepsilon \rightarrow 0$ we arrive at the statement II of the proposition.

5.2. Proof of Theorem 2. We will construct the family $f_{i}=f_{i}^{(\varepsilon)} \in$ $L_{2}(D), i=0,1, \ldots, N_{\varepsilon}$, where $N_{\varepsilon} \rightarrow \infty$ as $\varepsilon \rightarrow 0$, satisfying the assumptions $\mathbf{a}, \mathbf{b}, \mathbf{c}_{1}, \mathbf{c}_{2}$ of Proposition 7 .

Remind that in our problem $D=[0,1]^{d}$ and $\mathscr{F}=B_{\mathbf{p}, \infty}^{\mathbf{s}}(M)$ and $G(f)=f$ and $\|\cdot\|=\|\cdot\|_{p}$, where $\|\cdot\|_{p}$ is $L_{p}$-norm on $[0,1]^{d}$.

Let us fix some function $F: \mathbf{R} \rightarrow \mathbf{R}$ such that $\operatorname{supp}(F) \subset[-1,1]$, $\|F\|_{\infty} \leqslant 1, F \in H(\beta, 1)$, where $\beta=\max _{j=1, \ldots, d} s_{j}$ and $H(\beta, 1)$ is a Hölder ball.

Let us define $F_{d}: \mathbf{R}^{d} \rightarrow \mathbf{R}$ by $F_{d}(x)=\prod_{j=1}^{d} F\left(x_{j}\right), x=\left(x_{1}, \ldots\right.$, $\left.x_{d}\right) \in \mathbf{R}^{d}$.

Set $\delta=c \varepsilon \sqrt{\ln \varepsilon^{-1}}, h_{k}=\delta^{a_{k}}, k=1, \ldots, d, A_{\varepsilon}=\delta^{a}$, where the constant $c$ will be chosen later. Below, we will give explicit expressions for the real numbers $a>0$ and $a_{k}>0, k=1, \ldots, d$. Now, let us set $\widetilde{a}=\min _{k=1, \ldots, d} a_{k}$ and let $b>0$ be an arbitrary real number strictly less than $\widetilde{a}$. Set $M_{\varepsilon}=\varepsilon^{-b}$ (without loss of generality we will assume that $M_{\varepsilon}$ is an integer).

Let $\mathscr{B}:=\left\{u_{m}=i / M_{\varepsilon}, m=1, \ldots, M_{\varepsilon}-1,\right\}$ and let $\mathscr{B}^{d}=\otimes_{j=1}^{d} \mathscr{B}$. $\mathscr{B}^{d}$ is obviously a net in $[0,1]^{d}$ and $N_{\varepsilon}:=\operatorname{card}\left(\mathscr{B}^{d}\right)=\left(\varepsilon^{-b}-1\right)^{d}$. 
Finally, let us define $f_{i}:[0,1]^{d} \rightarrow \mathbf{R}, i=0,1, \ldots, N_{\varepsilon}$, as follows:

$$
\begin{gathered}
f_{0}(t) \equiv 0, \quad f_{i}(t)=M A_{\varepsilon} F_{d}\left(\frac{t-t_{i}}{h}\right), \quad t \in[0,1]^{d}, \quad t_{i} \in \mathscr{B}^{d}, \\
\text { where } h=\left(h_{1}, \ldots, h_{d}\right) .
\end{gathered}
$$

Let us make several remarks.

1. Clearly $N_{\varepsilon} \rightarrow \infty$ as $\varepsilon \rightarrow 0$ and the number $\ln N_{\varepsilon}$ which appears in the assumption $\mathbf{c}_{2}$ is $\ll \sim b d \ln \varepsilon^{-1} \gg$.

2. In view of the choice of the function $F$ and the net $\mathscr{B}^{d}$ we have for all $\varepsilon>0$ small enough $\left[f_{i}(t)-f_{0}(t)\right]\left[f_{j}(t)-f_{0}(t)\right] \equiv 0$ for all $i \neq j$, $i, j=1, \ldots, N_{\varepsilon}$. Thus, assumption $\mathbf{c}_{1}$ is fulfilled with $\mathfrak{M}=0$.

3 . In view of the choice of the family $f_{i}, i=0,1, \ldots, N_{\varepsilon}$, assumption $\mathbf{b}$ is fulfilled for all $\varepsilon>0$ small enough and we find that

$$
a_{\varepsilon}:=\left\|f_{i}-f_{0}\right\|_{p}=A_{\varepsilon}\left(\prod_{k=1}^{d} h_{k}\right)^{1 / p} M\left\|F_{d}\right\|_{p}=M\left\|F_{d}\right\|_{p} \delta^{\left(a+(1 / p) \sum_{k=1}^{d} a_{k}\right)} .
$$

4. In view of the choice of the function $F$ it is easy to see that assumption $\mathbf{a}$ is fulfilled (i.e., $\left.\left(f_{i}, i=1, \ldots, N_{\varepsilon}\right) \subset B_{\mathbf{p}, \infty}^{\mathbf{s}}(M)\right)$ if

$$
\frac{A_{\varepsilon}\left(\prod_{k=1}^{d} h_{k}\right)^{1 / p_{j}}}{h_{j}^{s_{j}}}=1 \quad \text { for all } j=1, \ldots, d .
$$

This leads to the following system of equations for the numbers $a>0$ and $a_{k}>0, k=1, \ldots, d: a+p_{j}^{-1} \sum_{k=1}^{d} a_{k}-a_{j} s_{j}=0$ for all $j=1, \ldots, d$.

5. Fix $\rho \in(0,1)$ and set $c=\sqrt{\rho b d} / M$. In view of the choice of the family $\left(f_{i}, i=0,1, \ldots, N_{\varepsilon}\right)$ we obtain for all $i=1, \ldots, N_{\varepsilon}$ and $\varepsilon>0$ small enough

$$
\left\|f_{i}-f_{0}\right\|_{2}^{2}=\left\|F_{d}\right\|_{2}^{2} M^{2} A_{\varepsilon}^{2} \prod_{k=1}^{d} h_{k} \leqslant M^{2} \delta^{2 a+\sum_{k=1}^{d} a_{k}} .
$$

If the real numbers $a>0$ and $a_{k}>0, k=1, \ldots, d$, satisfy $2 a+$ $\sum_{k=1}^{d} a_{k}=2$, then for all $i=1, \ldots, N_{\varepsilon}$ and $\varepsilon>0$ small enough

$$
\left\|f_{i}-f_{0}\right\|_{2}^{2} \leqslant M^{2} \delta^{2}=\rho b d \varepsilon^{2} \ln \varepsilon^{-1}=\rho \varepsilon^{2} \ln N_{\varepsilon} .
$$

Thus, assumption $\mathbf{c}_{2}$ is fulfilled.

It remains to find the real numbers $a>0$ and $a_{k}>0, k=1, \ldots, d$, in order to calculate the rate $a_{\varepsilon}$. To do this, one has to solve

$$
a+\frac{1}{p_{j}} \sum_{k=1}^{d} a_{k}-a_{j} s_{j}=0 \quad \text { for all } j=1, \ldots, d, \quad 2 a+\sum_{k=1}^{d} a_{k}=2 .
$$

The solution is

$$
a_{j}=2\left[\frac{1}{s_{j} p_{j}}+\frac{a}{s_{j}}\left(\frac{1}{2}-\frac{1}{p_{j}}\right)\right], \quad a=\frac{1-\sum_{j=1}^{d} 1 /\left(s_{j} p_{j}\right)}{1-\sum_{j=1}^{d}\left(1 / p_{j}-\frac{1}{2}\right)\left(1 / s_{j}\right)} .
$$

From here we obtain that $a+p^{-1} \sum_{k=1}^{d} a_{k}=K / L$ and hence $a_{\varepsilon}=$ $M\left\|F_{d}\right\|_{p} \delta^{K / L}$.

Finally, we find that $a_{\varepsilon} \asymp M^{(L-K) / L}\left(\varepsilon \sqrt{d \ln \varepsilon^{-1}}\right)^{K / L}$ as $\varepsilon \rightarrow 0$. 


\section{СПИСОК ЛИТЕРАТУРЫ}

1. Donoho D.L., Johnstone I.M., Kerkyacharian G., Picard D. Wavelet shrinkage: asymptopia? (With discussion and a reply by the authors.) - J. Roy. Statist. Soc., Ser. B, 1995, v. 57, № 2, p. 301-369.

2. Härdle W., Kerkyacharian G., Picard D., Tsybakov A. Wavelets, Approximation, and Statistical Applications. New York: Springer-Verlag, 1998, 265 p. (Lecture Notes in Statist., v. 129.)

3. Ибрагимов И.А., Хасминский Р. З. Оценка максимального значения сигнала в гауссовском белом шуме. - Матем. заметки, 1982, т. 32, № 4, с. 529-536.

4. Ingster Yu.I. Asymptotically minimax hypothesis testing for nonparametric alternatives. I, II, III. - Math. Methods Statist., 1993, v. 2, № 2, p. 85-114; № 3, p. 171-189; № 4, p. 249-268.

5. Kerkyacharian G., Lepski O., Picard D. Nonlinear estimation in anisotropic multiindex denoising. - Probab. Theory Related Fields, 2001, v. 121, № 2, p. 137-170.

6. Лепский $О . B$. Об одной задаче адаптивного оценивания в гауссовском белом шуме. - Теория вероятн. и ее примен., 1990 , т. 35 , в. 3 , с. 459-470.

7. Лепский О.В. Асимптотически минимаксное адаптивное оценивание. I: Верхние границы. Оптимально адаптивные оценки. - Теория вероятн. и ее примен., 1991, т. 36 , в. 4 , с. $645-659$.

8. Lepskii O. V. On problems of adaptive estimation in white Gaussian noise. - Adv. Soviet Math., 1992, v. 12, p. 87-106.

9. Лепский О.B. Об оценивании максимума непараметрического сигнала с точностью до постоянной. - Теория вероятн. и ее примен., 1993 , т. 38 , в. 1 , c. $187-194$.

10. Lepski O. V., Mammen E., Spokoiny V.G. Optimal spatial adaptation to inhomogeneous smoothness: an approach based on kernel estimates with variable bandwidth selectors. - Ann. Statist., 1997, v. 25, № 3, p. 929-947.

11. Lepski O. V., Tsybakov A.B. Asymptotically exact nonparametric hypothesis testing in sup-norm and at a fixed point. - Probab. Theory Related Fields, 2000, v. 117, № 1, p. 17-48.

12. Немировский $A$.C. Непараметрическое оценивание гладких функций регрессии. - Изв. АН СССР, сер. техн. кибернет., 1985, № 3, с. 50-60.

13. Никольский C. М. Приближение функций многих переменных и теоремы вложения. М.: Наука, 1977, 455 с.

14. Nussbaum $M$. Spline smoothing in regression models and asymptotic efficiency in $L_{2}$. - Ann. Statist., 1985, v. 13, № 3, p. 984-997.

15. Нуссбаум $M$. О непараметрическом оценивании функций регрессии, глацкой в области из $\mathbf{R}^{k}$. - Теория вероятн. и ее примен., 1986 , т. 31 , в. 1, с. $118-125$.

16. Walsh J.B. An introduction to stochastic partial differential equations. - Lecture Notes in Math., 1984, v. 1180, p. 265-439. 